This is the post peer-review accepted manuscript of:

K. Magowe, A. Giorgetti, S. Kandeepan and X. Yu, "Statistical distribution of position error in weighted centroid localization," IEEE International Conference on Communications (ICC), Paris, 2017, pp. 1-5.

The published version is available online at:

https://doi.org/10.1109/ICC.2017.7996836

(C) 2017 IEEE. Personal use of this material is permitted. Permission from IEEE must be obtained for all other uses, in any current or future media, including reprinting/republishing this material for advertising or promotional purposes, creating new collective works, for resale or redistribution to servers or lists, or reuse of any copyrighted component of this work in other works. 


\title{
Statistical Distribution of Position Error in Weighted Centroid Localization
}

\author{
Kagiso Magowe*, Andrea Giorgetti ${ }^{\dagger}$, Sithamparanathan Kandeepan*, and Xinghuo Yu* \\ ${ }^{\dagger}$ DEI, University of Bologna, Italy \\ ${ }^{*}$ School of Engineering, RMIT University, Melbourne, Australia \\ Email: kagiso.magowe.au@ieee.org
}

\begin{abstract}
Weighted centroid localization (WCL) based on received signal strength (RSS) measurements is an attractive lowcomplexity solution that enables cognitive radios (CRs) to have a geolocation awareness of the radio environment. In this paper, we propose a new analytical framework to accurately calculate the performance of WCL based on the statistical distribution of the ratio of two quadratic forms in normal variables. In particular, we derive an exact expression for the cumulative distribution function (CDF) of the two-dimensional location estimation in the presence of independent and identically distributed (i.i.d.) as well as correlated shadowing. Numerical results confirm that the analytical framework is able to predict the performance of WCL capturing all the essential aspects of propagation as well as CR network spatial topology.
\end{abstract}

\section{INTRODUCTION}

Cognitive radio (CR) is one of the emerging technologies that has been developed and studied over the past decade to enable efficient utilization of the spectrum resources [1]. In CR networks, geo-location of primary user (PU) is a spectrum awareness technique that not only plays an important role in preventing harmful interference to the PU, but allows for better spectrum resource allocations in the spatial domain [2]-[9]. Bearing in mind the challenges posed by the uncooperative nature of the PU, weighted centroid localization (WCL) represents an attractive low complexity solution which can rely only on e.g., received signal strength (RSS) measurements.

The original coarse-grained centroid localization algorithm used to estimate the position of the transmitter using only the coordinates of the receiving devices in an outdoor environment was proposed in [10]. Subsequently, the WCL technique has been studied in several papers assuming the secondary users (SUs) have limited information about the PU, under varying environment conditions, i.e., factors such as node placement, node density, shadowing variance and node spacing [10][17]. WCL algorithm in Zigbee sensor networks based on either RSS or link quality indicator was presented in [11]. Relative span weighted localization and relative span exponential weighted localization (REWL) mechanisms, which assign linear weights and exponential weights, respectively were introduced in [12].

Most of the literature analyze the WCL performance in terms of the root mean square error (RMSE), ignoring the statistical distribution of the error. However, in many practical situations e.g., in econometrics or engineering just studying moments of estimators is not sufficient and in some instances such moments do not exist [18]. Thus, one can consider the distribution function as a performance criterion, in which an estimator with high probabilities of being close to the true parameter is the most favorable [18], [19]. The first theoretical framework for WCL analysis using a probabilistic approach was presented in [13], assuming that the two-dimensional localization errors are jointly Gaussian, thus requiring the calculation of the error covariance matrix. Recently, an analytical framework to analyze WCL in the presence of interference has been proposed in [17], for the case where the cyclic autocorrelations of the received signals are used as weights. A closed-form expression for the RMSE of the two-dimensional location estimation for RSS-based WCL is presented in [20].

In this paper, we propose a new analytical framework to calculate the exact performance of WCL in the presence of independent and identically distributed (i.i.d.) as well as correlated log-normal shadowing, based on the statistical distribution of the ratio of two quadratic forms in normal variables [17], [18], [21]. In particular, we derive an expression for the cumulative distribution function (CDF) of the localization error based on the inversion formula for an indefinite quadratic form [18], [22], [23]. In summary, the main contributions of this paper are the following:

- We derive the exact expression for the CDF of the error of the two-dimensional location estimation.

- The exact expression is based on the statistical distribution of the ratio of quadratic forms in normal variables.

- The analytical framework includes the most general case of correlated shadowing and can be easily simplified to handle i.i.d. shadowing as well.

- Through the new analytical methodology we quantify the performance of WCL in different scenarios by varying the PU locations, the path-loss exponent, the shadowing parameter, the number of SUs, and their location.

- A key contribution in our analysis is that as well as presenting the exact performance in terms of the CDF, our analytical framework takes into consideration the finite nature of the network, overcoming the limitations of asymptotic results based on the central limit theorem.

Throughout the paper boldface letters denote matrices and 
vectors; $\mathbf{G} \sim \mathcal{N}(\boldsymbol{\mu}, \boldsymbol{\Sigma})$ denotes a Gaussian distributed vector with mean $\boldsymbol{\mu}$ and covariance matrix $\boldsymbol{\Sigma} ; \mathrm{F}(q)$ denotes the CDF of a random variable $Q ; \mathbf{I}$ is the identity matrix; $\mathbf{1}$ denotes a square matrix of ones; $(\cdot)^{\mathrm{T}}$ stands for transposition and $\|\cdot\|_{l}$ stands for the $l$-th norm.

The remainder of this paper is organized as follows. Section II presents the system model and the performance metric of WCL. Section III provides the analytical framework for the exact expression of the CDF of the estimation error. A case study to quantify the effectiveness of the proposed approach is analyzed in Section IV. Section V concludes the paper.

\section{SYSTEM MODEL}

We consider a CR network with $N$ SUs located in a square area of side length $\mathcal{S}$, and the PU located within the area at position $\mathbf{L}_{\mathrm{p}}=\left[x_{\mathrm{p}}, y_{\mathrm{p}}\right]^{\mathrm{T}}$. The position of the $i$-th SU node is defined as $\mathbf{L}_{i}=\left[x_{i}, y_{i}\right]^{\mathrm{T}}, i=1,2, \ldots, N$. The propagation environment is characterized by a power-law path-loss channel model plus log-normal shadowing. The RSS at the $i$-th SU node from the PU is thus given by

$$
P_{i}=P_{\mathrm{T}}-p_{0}\left(d_{0}\right)-10 \alpha \log _{10}\left(\frac{\left\|\mathbf{L}_{i}-\mathbf{L}_{\mathrm{p}}\right\|_{2}}{d_{0}}\right)+S_{i}
$$

where $P_{\mathrm{T}}$ is the transmit power in $\mathrm{dBm}, p_{0}\left(d_{0}\right)$ is the path-loss at a reference distance $d_{0}, \alpha$ is the path-loss exponent, and $S_{i}$ describes the random shadowing effect. More precisely, in the general case of correlated shadowing, $\mathbf{S}=\left[S_{1}, \ldots, S_{N}\right]^{\mathrm{T}} \sim$ $\mathcal{N}\left(\mathbf{0}, \boldsymbol{\Sigma}_{\mathrm{s}}\right)$ where $\boldsymbol{\Sigma}_{\mathrm{s}}$ is the shadowing covariance matrix. In the case of independent shadowing among sensors $\boldsymbol{\Sigma}_{\mathrm{s}}=$ $\operatorname{diag}\left(\sigma_{\mathrm{s}, 1}^{2}, \ldots, \sigma_{\mathrm{s}, N}^{2}\right)$, which further simplifies in the i.i.d. case to $\boldsymbol{\Sigma}_{\mathrm{s}}=\sigma_{\mathrm{s}}^{2} \mathbf{I}$. For the correlated shadowing case, we consider the following model for spatial dependency [24]-[26]

$$
\boldsymbol{\Sigma}_{\mathrm{s}}=\left[\Sigma_{i j}\right]_{i, j=1, \ldots, N} \quad \text { with } \quad \Sigma_{i j}=\sigma_{\mathrm{s}}^{2} e^{-\beta\left\|\mathbf{L}_{i}-\mathbf{L}_{j}\right\|_{2}}
$$

where $e^{-\beta}$ is the correlation coefficient at $1 \mathrm{~m}$, while $1 / \beta=$ $D_{\text {c }}$ is the correlation distance in meters.

We begin by presenting the WCL algorithm used to estimate the location of the PU in two dimensions [11]

$$
\widehat{\mathbf{L}}_{\mathrm{p}}=\frac{\sum_{i=1}^{N} w_{i} \mathbf{L}_{i}}{\sum_{i=1}^{N} w_{i}}=\frac{\sum_{i=1}^{N}\left(P_{i}-P_{\min }\right) \mathbf{L}_{i}}{\sum_{i=1}^{N}\left(P_{i}-P_{\min }\right)}
$$

where $w_{i}=\left(P_{i}-P_{\min }\right) /\left(P_{\max }-P_{\min }\right)$ is the weighting coefficient for the $i$-th SU node, $P_{\max }$ is the maximum received power among sensor nodes, and $P_{\min }$ is an arbitrary reference power level which can be e.g., the minimum measurable received power by the SU. The localization error is defined as $\boldsymbol{\xi} \triangleq \widehat{\mathbf{L}}_{\mathrm{p}}-\mathbf{L}_{\mathrm{p}}=\left[\widehat{X}_{\mathrm{p}}-x_{\mathrm{p}}, \widehat{Y}_{\mathrm{p}}-y_{\mathrm{p}}\right]^{\mathrm{T}}$, where $\widehat{X}_{p}$ and $\widehat{Y}_{p}$ are the one-dimensional position estimates along the $\mathrm{x}$-axis and $\mathrm{y}$-axis, respectively

$$
\widehat{X}_{p}=\frac{\sum_{i=1}^{N} G_{i} x_{i}}{\sum_{i=1}^{N} G_{i}} \quad \widehat{Y}_{p}=\frac{\sum_{j=1}^{N} G_{j} y_{j}}{\sum_{j=1}^{N} G_{j}}
$$

with $G_{i}=P_{i}-P_{\min }$. Finally, the squared distance error is given by

$$
\xi^{2} \triangleq\left(\widehat{X}_{\mathrm{p}}-x_{\mathrm{p}}\right)^{2}+\left(\widehat{Y}_{\mathrm{p}}-y_{\mathrm{p}}\right)^{2}=\|\boldsymbol{\xi}\|_{2}^{2}
$$

For notational convenience we define $\mathbf{G}=\left[G_{1}, \ldots, G_{N}\right]^{\mathrm{T}}$, $\mathbf{X}=\left[x_{1}, \ldots, x_{N}\right]^{\mathrm{T}}$, and $\mathbf{Y}=\left[y_{1}, \ldots, y_{N}\right]^{\mathrm{T}}$. In the following section, the square of the distance error (4) will be interpreted as the ratio of two quadratic forms, leading to a new theoretical framework for the performance analysis of WCL.

\section{Cumulative Distribution Function of The LOCALIZATION ERROR}

In this section we derive the CDF expression of the error of the two-dimensional location estimate. Defining $\xi_{\mathrm{x}}=\widehat{X}_{\mathrm{p}}-x_{\mathrm{p}}$ and $\xi_{\mathrm{y}}=\widehat{Y}_{\mathrm{p}}-y_{\mathrm{p}}$ as the errors in the x-dimension and $\mathrm{y}$ dimension, respectively, the squared error can be written as

$$
\begin{aligned}
\xi^{2} & =\xi_{\mathrm{x}}^{2}+\xi_{\mathrm{y}}^{2} \\
& =\left(\frac{\sum_{i=1}^{N} G_{i}\left(x_{i}-x_{\mathrm{p}}\right)}{\sum_{i=1}^{N} G_{i}}\right)^{2}+\left(\frac{\sum_{i=1}^{N} G_{j}\left(y_{j}-y_{\mathrm{p}}\right)}{\sum_{i=1}^{N} G_{j}}\right)^{2} \\
& =\frac{\sum_{i=1}^{N} \sum_{j=1}^{N} G_{i} G_{j} a_{i j}}{\sum_{i=1}^{N} \sum_{j=1}^{N} G_{i} G_{j}}
\end{aligned}
$$

where $a_{i j}=\left(x_{i}-x_{\mathrm{p}}\right)\left(x_{j}-x_{\mathrm{p}}\right)+\left(y_{i}-y_{\mathrm{p}}\right)\left(y_{j}-y_{\mathrm{p}}\right)$. Defining $x_{i}^{\prime}=x_{i}-x_{\mathrm{p}}$ and $y_{i}^{\prime}=y_{i}-y_{\mathrm{p}}$, the term $a_{i j}$ can be expressed as $a_{i j}=x_{i}^{\prime} x_{j}^{\prime}+y_{i}^{\prime} y_{j}^{\prime}$, and arranged in a matrix form $\mathbf{A}=\left[a_{i, j}\right]_{i, j=1, \ldots, N}$ with $\mathbf{A}=\mathbf{X}^{\prime} \mathbf{X}^{\prime \mathrm{T}}+\mathbf{Y}^{\prime} \mathbf{Y}^{\prime \mathrm{T}}$, $\mathbf{X}^{\prime}=\left[x_{1}^{\prime}, x_{2}^{\prime}, \ldots, x_{N}^{\prime}\right]^{\mathrm{T}}$, and $\mathbf{Y}^{\prime}=\left[y_{1}^{\prime}, y_{2}^{\prime}, \ldots, y_{N}^{\prime}\right]^{\mathrm{T}}$. It should be noted that $\mathbf{A}$ is symmetric. Now, using matrix-vector notation we obtain the following compact form of the squared error

$$
\xi^{2}=\frac{\mathbf{G}^{\mathrm{T}} \mathbf{A G}}{\mathbf{G}^{\mathrm{T}} \mathbf{B G}}
$$

with $\mathbf{G} \sim \mathcal{N}\left(\boldsymbol{\mu}, \boldsymbol{\Sigma}_{\mathrm{s}}\right), \boldsymbol{\mu}=\mathbb{E}[\mathbf{G}]$, i.e., $\boldsymbol{\mu}=\left[\mu_{1}, \ldots, \mu_{N}\right]^{\mathrm{T}}$ with $\mu_{i}=\mathbb{E}\left[G_{i}\right]=\mathbb{E}\left[P_{i}\right]-P_{\min }$ and $\mathbf{B}=\mathbf{1}$.

The CDF of the error $\xi, F\left(q_{0}\right)=\operatorname{Pr}\left[\xi \leq q_{0}\right]$, known as localization error probability (LEP), can be derived as follows, noting first that $\operatorname{Pr}\left[\xi \leq q_{0}\right]=\operatorname{Pr}\left[\xi^{2} \leq q_{0}^{2}\right]$, [18]

$$
\begin{aligned}
\mathbf{F}\left(q_{0}\right) & =\operatorname{Pr}\left[\xi \leq q_{0}\right] \\
& =\operatorname{Pr}\left[\frac{\mathbf{G}^{\mathrm{T}} \mathbf{A} \mathbf{G}}{\mathbf{G}^{\mathrm{T}} \mathbf{B} \mathbf{G}} \leq q_{0}^{2}\right] \\
& =\operatorname{Pr}\left[\mathbf{G}^{\mathrm{T}} \mathbf{A} \mathbf{G} \leq q_{0}^{2} \mathbf{G}^{\mathrm{T}} \mathbf{B} \mathbf{G}\right] \\
& =\operatorname{Pr}\left[\mathbf{G}^{\mathrm{T}} \mathbf{W} \mathbf{G} \leq 0\right]
\end{aligned}
$$

where $\mathbf{W}=\mathbf{A}-q_{0}^{2} \mathbf{B}$. The expression (6) shows that the distribution of the ratio of quadratic forms reduces to the distribution of an indefinite quadratic form $\mathbf{G}^{\mathrm{T}} \mathbf{W G}$, where $\mathbf{W}$ is an indefinite matrix. Using $\boldsymbol{\Sigma}_{\mathrm{s}}$, we can also perform a whitening of the vector $\mathbf{G}$ which allows to rewrite (6) in the following form 


$$
\begin{aligned}
\mathbf{F}\left(q_{0}\right) & =\operatorname{Pr}\left[\mathbf{G}^{\mathrm{T}} \boldsymbol{\Sigma}_{\mathrm{s}}{ }^{-1 / 2} \boldsymbol{\Sigma}_{\mathbf{s}}{ }^{1 / 2} \mathbf{W} \boldsymbol{\Sigma}_{\mathrm{s}}{ }^{1 / 2} \boldsymbol{\Sigma}_{\mathrm{s}}{ }^{-1 / 2} \mathbf{G} \leq 0\right] \\
& =\operatorname{Pr}\left[\mathbf{G}^{\mathrm{T}} \mathbf{V}^{-1} \mathbf{P} \mathbf{P}^{\mathrm{T}} \mathbf{V} \mathbf{W} \mathbf{V} \mathbf{P P}^{\mathrm{T}} \mathbf{V}^{-1} \mathbf{G} \leq 0\right] \\
& =\operatorname{Pr}\left[\mathbf{Z}^{\mathrm{T}} \boldsymbol{\Lambda} \mathbf{Z} \leq 0\right]
\end{aligned}
$$

where $\mathbf{V}=\boldsymbol{\Sigma}_{\mathbf{s}}{ }^{1 / 2}, \mathbf{Z}=\mathbf{P}^{\mathrm{T}} \mathbf{V}^{-1} \mathbf{G}, \mathbf{P}$ is an orthogonal matrix formed by the eigenvectors of $\mathbf{V W V}$, and $\boldsymbol{\Lambda}=\mathbf{P}^{\mathrm{T}} \mathbf{V W V P}$ is a diagonal matrix containing the eigenvalues $\lambda_{1}, \ldots, \lambda_{N}$ of VWV.

Note now that $\mathbf{Z} \sim \mathcal{N}\left(\boldsymbol{\mu}_{z}, \mathbf{I}\right)$ with $\boldsymbol{\mu}_{z}=\mathbf{P}^{\mathrm{T}} \mathbf{V}^{-1} \boldsymbol{\mu}$, so the expression (7) can be casted into

$$
\mathrm{F}\left(q_{0}\right)=\operatorname{Pr}\left[\sum_{i=1}^{N} \lambda_{i} z_{i}^{2} \leq 0\right]
$$

where $z_{i}^{2} \sim \chi^{2}\left(\mu_{z i}^{2}\right)$ is a noncentral chi-squared distributed random variable with one degree of freedom and noncentral parameter $\mu_{z i}^{2}{ }^{1}$ A numerical integration representation of (8) was presented by Imhof [23], which is based on the Gil-Pelaez inversion formula [22]

$$
\mathrm{F}\left(q_{0}\right)=\frac{1}{2}-\frac{1}{\pi} \int_{0}^{\infty} t^{-1} \Im\{\phi(t)\} d t
$$

where $\Im\{\cdot\}$ represents the imaginary part and

$$
\phi(t)=\left[\prod_{j=1}^{N}\left(1-2 i t \lambda_{j}\right)^{-1 / 2}\right] \exp \left(i \sum_{j=1}^{N} \frac{\mu_{z j}^{2} \lambda_{j} t}{1-2 i t \lambda_{j}}\right)
$$

is the characteristic function of $\mathbf{Z}^{\mathrm{T}} \boldsymbol{\Lambda} \mathbf{Z}$. Alternatively, in [23], it has been shown that (9) can be written as

$$
\mathrm{F}\left(q_{0}\right)=\frac{1}{2}-\frac{1}{\pi} \int_{0}^{\infty} \frac{\sin \theta(v)}{v \rho(v)} d v
$$

where

$$
\begin{gathered}
\theta(v)=\frac{1}{2} \sum_{j=1}^{N}\left(r_{j} \tan ^{-1}\left(\lambda_{j} v\right)+\mu_{z j}^{2} \lambda_{j} v\left(1+\lambda_{j}^{2} v^{2}\right)^{-1}\right) \\
\rho(v)=\left[\prod_{j=1}^{N}\left(1+\lambda_{j}^{2} v^{2}\right)^{r_{j} / 4}\right] \exp \left(\frac{1}{2} \sum_{j=1}^{N} \frac{\mu_{z j}^{2} \lambda_{j}^{2} v^{2}}{1+\lambda_{j}^{2} v^{2}}\right)
\end{gathered}
$$

and $r_{j}$ 's are the multiplicities of the nonzero distinct $\lambda_{j}$ 's. We remark that both (9) and (10) provide the same accuracy, however, the latter does not involve the use of complex numbers.

\footnotetext{
${ }^{1}$ Note that for the i.i.d. shadow fading case $\mathbf{Z} \sim \mathcal{N}\left(\boldsymbol{\mu}_{z}, \mathbf{I}\right)$, with $\boldsymbol{\mu}_{z}=$ $\mathbf{P}^{\mathrm{T}} \boldsymbol{\mu} / \sigma_{\mathrm{s}}$ and $\mathbf{V}^{-1}=\mathbf{I} / \sigma_{\mathrm{s}}$.
}

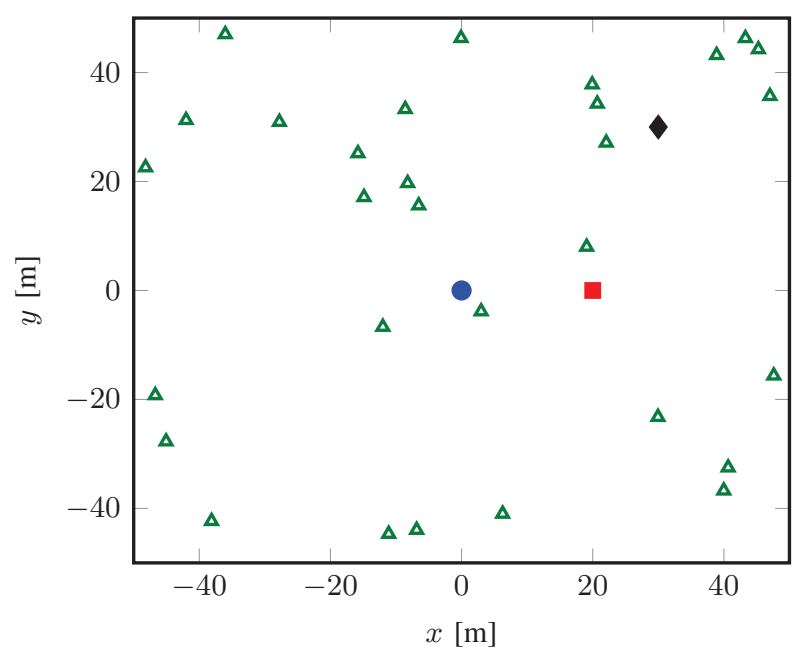

Fig. 1. The case study considered with randomly located SUs (triangles) and different PU positions: circle, square and diamond represent locations $\mathbf{L}_{\mathrm{p}}^{\mathrm{A}}, \mathbf{L}_{\mathrm{p}}^{\mathrm{B}}$ and $\mathbf{L}_{\mathrm{p}}^{\mathrm{C}}$, respectively.

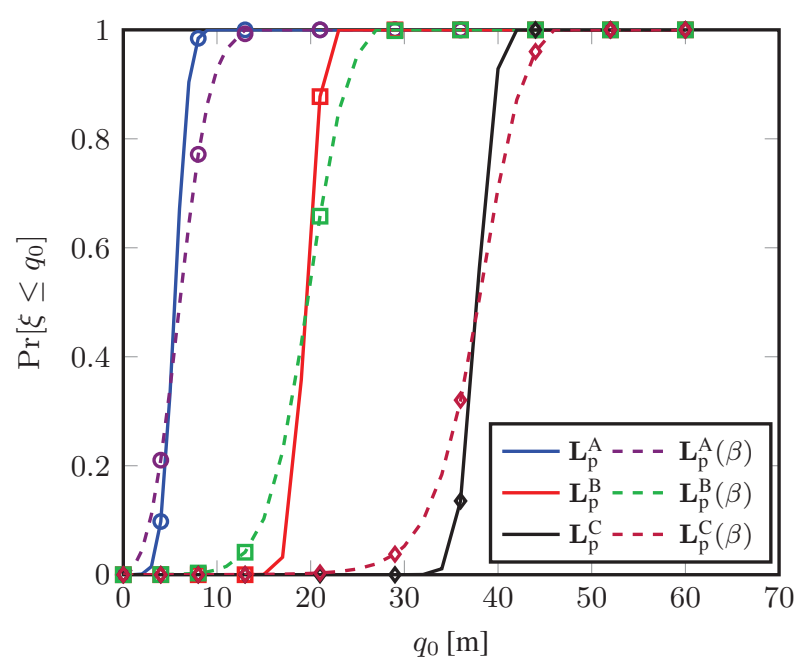

Fig. 2. CDF of the error of the two-dimensional position estimation, in the RF-SU case, when the PU is located at $\mathbf{L}_{\mathrm{p}}^{\mathrm{A}}, \mathbf{L}_{\mathrm{p}}^{\mathrm{B}}$ and $\mathbf{L}_{\mathrm{p}}^{\mathrm{C}}$, for $N=50$. Dashed lines refer to correlated shadowing with $\beta=1 / 30 \mathrm{~m}^{-1}$.

\section{Case Study Analysis}

In this section, we exploit the methodology provided in Section III to analyze the performance of WCL in terms of the LEP, $\mathrm{F}\left(q_{0}\right)=\operatorname{Pr}\left[\xi \leq q_{0}\right]$. In particular, we compare analytical and Monte-Carlo simulation results of the CDF of location estimation in two different settings: 1) random, but fixed, SU (RF-SU) positions, and 2) random SU (R-SU) positions, respectively. ${ }^{2}$

${ }^{2}$ For random-fixed SUs, we consider a single snapshot of SU positions while for random SU positions we average the analytical and simulation results over 1000 independent realizations. 




Fig. 3. The CDF of the error of the two-dimensional position estimation, in the R-SU case, when the PU is located at $\mathbf{L}_{\mathrm{p}}^{\mathrm{A}}, \mathbf{L}_{\mathrm{p}}^{\mathrm{B}}$ and $\mathbf{L}_{\mathrm{p}}^{\mathrm{C}}$, for $N=10$ (solid lines) and $N=50$ (dashed lines).

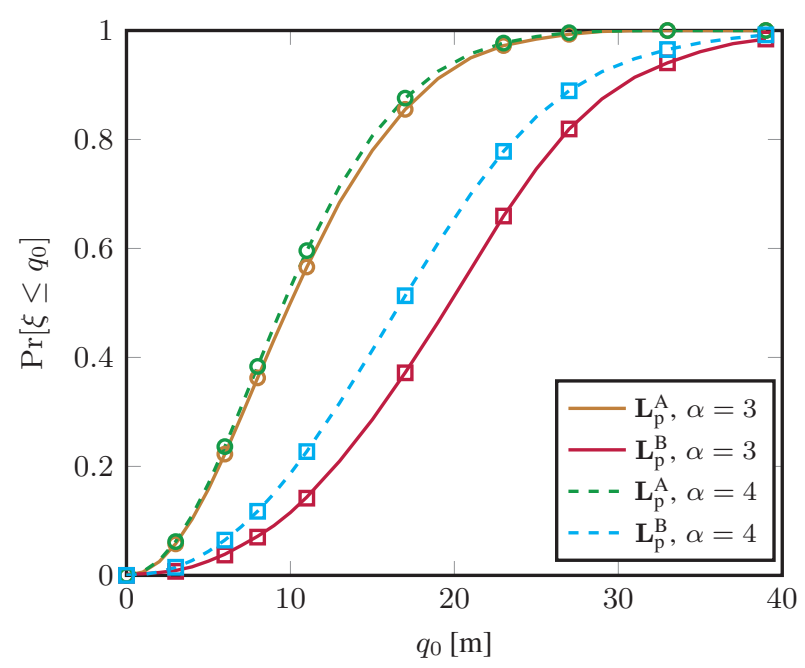

Fig. 4. CDF of the error of the two-dimensional position estimation, in the R-SU case, when the PU is located at $\mathbf{L}_{\mathrm{p}}^{\mathrm{A}}$ and $\mathbf{L}_{\mathrm{p}}^{\mathrm{B}}$, and for two different values of the path-loss exponent, with $N=10$.

The case study scenario is a square area with side $\mathcal{S}=$ $100 \mathrm{~m}$. Fig. 1 depicts the considered randomly distributed SUs and the PU located in three different positions: $\mathbf{L}_{\mathrm{p}}^{\mathrm{A}}=$ $(0 \mathrm{~m}, 0 \mathrm{~m}), \mathbf{L}_{\mathrm{p}}^{\mathrm{B}}=(20 \mathrm{~m}, 0 \mathrm{~m})$ and $\mathbf{L}_{\mathrm{p}}^{\mathrm{C}}=(30 \mathrm{~m}, 30 \mathrm{~m})$.

The propagation environment is characterized by (1), where the PU transmit power is $P_{\mathrm{T}}=20 \mathrm{dBW}$, the shadowing parameter is $\sigma_{\mathrm{s}}=4 \mathrm{~dB}, 5.5 \mathrm{~dB}$ and $8 \mathrm{~dB}$, the path-loss at reference distance, $d_{0}=1 \mathrm{~m}$, is $p_{0}\left(d_{0}\right)=50 \mathrm{~dB}$ and the pathloss exponent is $\alpha=3$ and $\alpha=4$.

To validate the analytical approach we used Monte-Carlo simulation with $10^{6}$ runs. In all figures, lines and symbols refer to analytical and simulation results, respectively. The discussion of our numerical results will be based on the following effects: PU location, shadowing and path-loss. Throughout this



Fig. 5. CDF of the error of the two-dimensional position estimation, in the R-SU case, when the PU is located at $\mathbf{L}_{\mathrm{p}}^{\mathrm{A}}$ and $\mathbf{L}_{\mathrm{p}}^{\mathrm{B}}$, and for two different values of shadowing parameter, with $N=10$.

section, unless specified otherwise, the case study analysis will be based on the i.i.d. shadowing assumption. Fig. 2 - Fig. 5 summarize our CDF analysis when considering the RF-SU and R-SU positions.

Impact of PU location and correlated shadowing. In Fig. 2 we show the relationship of the CDF using the exact analytical expression (9) and Monte-Carlo simulation results, for different PU locations in the RF-SU case, $N=50, \alpha=4$, and correlated shadowing with $\sigma_{\mathrm{s}}=8 \mathrm{~dB}$ and $\beta=1 / 30 \mathrm{~m}^{-1}$. The curve that refers to $\mathrm{PU}$ position $\mathrm{L}_{\mathrm{p}}^{\mathrm{A}}$ reaches a high probability faster than the other curves, essentially confirming that the most favorable location scenario is when the PU is in the center.

We also investigated the effect of correlated shadowing and it appears that there are parts of the curves where correlation improves the performance. Interestingly, for $q_{0}=6 \mathrm{~m}, q_{0}=$ $28 \mathrm{~m}$ and $q_{0}=36 \mathrm{~m}$ the LEP is independent of correlation distance for locations $\mathbf{L}_{\mathrm{p}}^{\mathrm{A}}, \mathbf{L}_{\mathrm{p}}^{\mathrm{B}}$ and $\mathbf{L}_{\mathrm{p}}^{\mathrm{C}}$, respectively.

In Fig. 3 we provide results for a scenario similar to the one considered in Fig. 2, except that now performances are averaged over R-SU positions, and we also introduce results for $N=10$. The node density appears to improve the performance of the WCL, especially for high LEPs. However, for locations $\mathbf{L}_{\mathrm{p}}^{\mathrm{B}}$ and $\mathbf{L}_{\mathrm{p}}^{\mathrm{C}}$ we observe a crossing point between LEP curves at $q_{0}=13 \mathrm{~m}$ and $q_{0}=30 \mathrm{~m}$, respectively.

Path-loss analysis. In Fig. 4 we show the impact the variation of the path-loss exponent $\alpha$ has on the $\mathrm{CDF}$, and we considered the following scenarios: PU locations $\mathbf{L}_{\mathrm{p}}^{\mathrm{A}}$ and $\mathbf{L}_{\mathrm{p}}^{\mathrm{B}}$, R-SU setting, $\alpha=3$ and $4, \sigma_{\mathrm{s}}=5.5 \mathrm{~dB}$. The LEP improves with an increase in $\alpha$ since this induces a node selection strategy, effectively reducing the impact of the SUs with low RSS on location estimate [14], [16]. For $\mathbf{L}_{\mathrm{p}}^{\mathrm{A}}$ scenario, there is lack of dependency on $\alpha$ as it can be observed from the tightness of the CDF curves. However, for $\mathbf{L}_{\mathrm{p}}^{\mathrm{B}}$ scenario, the 
impact of $\alpha$ is apparent and the estimation is more senstive to this parameter.

Shadowing analysis. In Fig. 5 we show the impact the variation of the shadowing parameter $\sigma_{\mathrm{s}}$ has on the $\mathrm{CDF}$, and we considered $\sigma_{\mathrm{s}}=4 \mathrm{~dB}, \sigma_{\mathrm{s}}=8 \mathrm{~dB}$ and $\alpha=4$. Interestingly, as depicted, the CDF is not so senstive to $\sigma_{\mathrm{s}}$ as reflected by the tightness of the curves for variation in $\sigma_{\mathrm{s}}$.

\section{CONCLUSiOn}

In this paper, we proposed a new analytical framework to calculate the accurate performance of WCL in the presence of i.i.d. as well as correlated log-normal shadowing, based on the statistical distribution of the ratio of two quadratic forms in normal variables. In particular, we derived an exact CDF of the two-dimensional localization error. A case study analysis was performed to evaluate the accuracy of the proposed methodology. Specifically, we analyzed the performance of WCL under varying PU location, path-loss exponent, shadowing standard deviation, number of SUs and their location. Results confirm that the statistical framework is able to predict the performance of WCL very accurately, capturing all the essential aspects of propagation, SUs location as well as the finite nature of the network.

\section{ACKNOWLEDGMENT}

This work was supported in part by the European Project EuroCPS through the H2020 Framework under Grant 644090, and the Australian Government Research Training Program Scholarship.

\section{REFERENCES}

[1] K. Sithamparanathan and A. Giorgetti, Cognitive Radio Techniques. Spectrum Sensing, Interference Mitigation and Localization. Boston, USA: Artech House Publishers, November 2012.

[2] A. Mariani, S. Kandeepan, and A. Giorgetti, "Periodic spectrum sensing with non-continuous primary user transmissions," IEEE Trans. on Wireless Comm., vol. 14, no. 3, pp. 1636-1649, March 2015.

[3] S. Yarkan and H. Arslan, "Exploiting location awareness toward improved wireless system design in cognitive radio," IEEE Commun. Mag., vol. 46, no. 1 , pp. 128-136, January 2008.

[4] S. Haykin, "Cognitive radio: brain-empowered wireless communications," IEEE J. Sel. Areas Commun., vol. 23, no. 2, pp. 201-220, February 2005.

[5] S. Bartoletti, W. Dai, A. Conti, and M. Z. Win, "A mathematical mode for wideband ranging," IEEE J. Sel. Topics Signal Process., vol. 9, no. 2 pp. 216-228, 2015.

[6] A. Conti, M. Guerra, D. Dardari, N. Decarli, and M. Z. Win, "Network experimentation for cooperative localization," IEEE J. Sel. Areas Commun., vol. 30, no. 2, pp. 467-475, 2012.

[7] M. Z. Win, A. Conti, S. Mazuelas, Y. Shen, W. M. Gifford, D. Dardari, and M. Chiani, "Network localization and navigation via cooperation," IEEE Commun. Mag., vol. 49, no. 5, pp. 56-62, 2011.
[8] W. Dai, Y. Shen, and M. Z. Win, "Energy-efficient network navigation algorithms," IEEE J. Sel. Areas Commun., vol. 33, no. 7, pp. 1418-1430, 2015.

[9] A. Conti, D. Dardari, M. Guerra, L. Mucchi, and M. Z. Win, "Experimental characterization of diversity navigation," IEEE Syst. J., vol. 8, no. 1, pp. 115-124, 2014.

[10] N. Bulusu, J. Heidemann, and D. Estrin, "Gps-less low-cost outdoor localization for very small devices," IEEE Personal Commun. Mag., vol. 7, no. 5, pp. 28-34, October 2000.

[11] J. Blumenthal, R. Grossmann, F. Golatowski, and D. Timmermann, "Weighted centroid localization in zigbee-based sensor networks," in Proc. IEEE Int. Symp. on Intelligent Signal Proc. (WISP), vol. 5, Alcala de Henares, SPAIN, October 2007, pp. 1-6.

[12] C. Laurendeau and M. Barbeau, "Centroid localization of uncooperative nodes in wireless networks using a relative span weighting method," EURASIP J. on Wireless Comm. and Netw., vol. 2010, pp. 1-10, November 2009.

[13] J. Wang, P. Urriza, Y. Han, and D. Cabric, "Weighted centroid localization algorithm: theoretical analysis and distributed implementation," IEEE Trans. Wireless Commun., vol. 10, no. 10, pp. 3403-3413, October 2011.

[14] A. Mariani, S. Kandeepan, A. Giorgetti, and M. Chiani, "Cooperative weighted centroid localization for cognitive radio networks," in Proc. IEEE Int. Symp. on Comm. and Info. Tech. (ISCIT), Gold Coast, AUSTRALIA, October 2012, pp. 459-464.

[15] K. Magowe and S. Kandeepan, "Cooperative blind localization of primary user in a cognitive radio environment," in Proc. IEEE Int. Conf. on Sig. Proc. and Comm. Sys. (ICSPCS), Gold Coast, AUSTRALIA, December 2014, pp. 1-8.

[16] K. Magowe, S. Kandeepan, A. Giorgetti, and X. Yu, "Constrained cluster based blind localization of primary user for cognitive radio networks," in Proc. IEEE Int. Symp. on Personal, Indoor, and Mobile Radio Comm. (PIMRC), Hong Kong, CHINA, September 2015, pp. 986-991.

[17] S. Chaudhari and D. Cabric, "Cyclic weighted centroid algorithm for transmitter localization in the presence of interference," IEEE Trans. on Cogn. Commun. Netw., vol. 2, no. 2, pp. 162-177, June 2016.

[18] A. Ullah, Finite sample econometrics. Oxford University Press, 2004.

[19] E. C. Chan and G. Baciu, Introduction to Wireless Localization: With IPhone SDK Examples. John Wiley \& Sons, 2012.

[20] A. Giorgetti, K. Magowe, and S. Kandeepan, "Exact analysis of weighted centroid localization," in Proc. European Sig. Pro. Conf. (EUSIPCO), vol. 24, Budapest, HUNGARY, August 2016, pp. 743-747.

[21] Y. Bao and R. Kan, "On the moments of ratios of quadratic forms in normal random variables," ELSEVIER J. of Multivariate Analysis, vol. 117, pp. 229-245, January 2015.

[22] J. Gil-Pelaez, "Note on the inversion theorem," Biometrika, vol. 38, no. 3-4, pp. 481-482, 1951.

[23] J. Imhof, "Computing the distribution of quadratic forms in normal variables," JSTOR Biometrika, vol. 48, no. 3/4, pp. 419-426, December 1961.

[24] M. Gudmundson, "Correlation model for shadow fading in mobile radio systems," Electron. Lett., vol. 27, no. 23, pp. 2145-2146, November 1991.

[25] D. Giancristofaro, "Correlation model for shadow fading in mobile radio channels," Electron. Lett., vol. 32, no. 11, pp. 958-959, February 1996.

[26] H. Claussen, "Efficient modelling of channel maps with correlated shadow fading in mobile radio systems," in Proc. IEEE Int. Symp. on Personal, Indoor, and Mobile Radio Comm. (PIMRC), vol. 4, Berlin, GERMANY, September 2005, pp. 512-516. 